\title{
The Development of the Dementia Screening Battery-100: Instrument Presentation, Reliability, and Construct Validity
}

\author{
Tarek Bellaj $^{a}$ Sonia Ben Jemaa ${ }^{b} \quad$ Maher Khelifa $^{a} \quad$ Mona Ben Djebara ${ }^{c}$ \\ Riadh Gouider $^{c}$ Didier Le Gall ${ }^{d}$ \\ a Psychology Program, Department of Social Sciences, College of Arts and Sciences, \\ Qatar University, Doha, Qatar; ${ }^{b}$ Faculty of Human and Social Sciences of Tunis, \\ Tunis University, Tunis, Tunisia; ' Neurology Department, Razi Hospital, Tunis, Tunisia; \\ ${ }^{\mathrm{d}}$ Laboratoire de Psychologie des Pays de la Loire, University of Angers, \\ Bretagne-Loire University, Angers, France
}

\section{Keywords}

Screening test - Neurodegenerative diseases - Elderly population · Developing countries ·

Tunisia $\cdot$ Non-Western cultures · Validation · Neuropsychological assessment

\begin{abstract}
Background/Aim: The screening of dementia in non-Westerners has so far relied on translations and adaptations of reputed instruments. Other efforts focused on developing culturally appropriate tests or tests in touch with new developments in the field. This study presents the rationale behind the construction of a new dementia screening test: the Dementia Screening Battery-100 (DSB-100). Methods: The DSB-100 was administered to 46 demented individuals and 159 healthy matched controls. All demented participants met the DSM-IV criteria for dementia. The healthy controls showed no cognitive impairment and were independent in activities of daily living. The DSB-100 was administered as part of a larger neuropsychological assessment to collect additional indices on the severity of patients' dementia, depression, and frontal dysfunctions. The same information was used for comparisons with DSB-100 scores. Results: Multiple regression analysis suggested that age and education, but not the variable sex, are essential in predicting cognitive performance. Construct validation yielded 4 factors, namely attention-visuospatial factors, memory, language, and executive functions. The results showed that the DSB-100 has a high interrater reliability and an acceptable overall internal homogeneity. Conclusion: These results validate the DSB-100 and suggest its appropriateness for dementia screening in Tunisian elderly and possibly elderly people from other cultures with modifications to some subscales.


Bellaj et al.: Dementia Screening Battery-100

\section{Introduction}

Among the neurological syndromes, dementia is one of the most complex due to diagnostic difficulties, especially in its early stages. Dementia is accompanied by cognitive deficits affecting different functions. Examining these functions through neuropsychological testing is necessary to document deficits directly that define dementia itself. With the emergence of the diagnostic entity "mild cognitive impairment" (MCI) with single or multiple domain impairment, this assessment becomes all the more useful in the absence of other efficient markers to distinguish these deficits or their progression [1]. For this purpose, it is important to distinguish between formal neuropsychological assessment, which is quite long and rich, and screening tools, which quickly yield the different levels of impairment.

Other than a short assessment time, screening tools must be simple to score, easily acceptable to patients, and should not require special or heavy equipment. They also need to be objective, quantitative, balanced between sensitivity and specificity, and have to satisfy classical psychometric qualities such as validity and reliability. Most importantly, they must be normalized and culturally appropriate for the population they assess. Hence, there is a real need for new screening tools that take into consideration and deal with all existing methodological limitations. It is especially important to address the many conceptual issues related to the universality of cognitive systems [2], the relationship between culture and cognition [3], the importance of demographic specificities of target populations [4], and cultural bias in psychological assessments [5].

Therefore, when we had to choose between translating, adapting, or developing a new instrument to assess dementia in Tunisia, we opted for the last to address 2 main concerns in existing measures in addition to the well-documented cultural and methodological issues [6, 7]. The first relates to methodological, conceptual, and ethical issues with available procedures and tests. The second relates to keeping up with rapidly changing knowledge in the field of dementia and its neuropsychological assessment.

As far as the first issue is concerned, typically any translation or adaptation includes serious problems of equivalence. The final product of any translation or adaptation inevitably includes equivalence bias and rarely equates with the original theoretical construct, administration method, and item content [8]. For instance, even when we are attentive to the procedures of translation and back-translation, the equivalence of tasks is always far from guaranteed. But avoiding verbal tests as a way to deal with bias is also not a solution, given that a large part of human cognitive activities are mediated by language and because non-verbal tests do not necessarily mean "culture-free tests" [9]. For translated and adapted tests, choosing items and pictures from local normalized databases - similar to Battig et al.'s category word frequency data [10] and Snodgrass and Vanderwart's norms for name agreement, familiarity, and visual complexity for pictures [11] - is not always appropriate, since scores that are "numerically identical can have a psychologically different meaning across cultures" [12]. This is especially true for adaptation procedures where inferences drawn from the adapted version scores can prove problematic as far as the psychological constructs are concerned [13]. This establishes the need to re-examine the construct validity of any adapted test, even if the items are based on standardized local databases.

Regarding the second concern, we noted a growing dissatisfaction with the increasing knowledge gap between the rapidly changing knowledge in the field of dementia and the existing dementia screening tools. Indeed, the most widely used instruments for the detection of dementia suffer from the following major limitations that our proposed instrument seeks to address:

- Most existing dementia screening instruments assess orientation in time and space, a complex function not altered in the early stages of dementia. This affects the discrimi- 
natory power of the measures, as they allot many points to such non-sensitive tasks for the early detection of dementia. This is the case for the Mini-Mental State Examination (MMSE) [14], the Montreal Cognitive Assessment (MoCA) [15], the Mattis Dementia Rating Scale (Mattis) [16], the Short Portable Mental Status Questionnaire [17], the modified MMSE (3MS) [18], the Addenbrooke's Cognitive Examination Revised [19], the Neurobehavioral Cognitive Status Examination (NCSE) [20], and the CERAD Neuropsychological Instrument [21].

- Several dementia screening instruments assess memory inappropriately using infraspan tasks (less than 6-7 units), even though long-term episodic memory deficit is a major criterion in the diagnosis of dementia according to the DSM-IV [22]. This is the case for the MMSE, 3MS, Mini-Cog, [23], MoCA, and for the Rowland Universal Dementia Assessment Scale (RUDAS) [24]. For example, registration and recall in the MMSE can be considered weak measures of memory, as each of the tasks used is far less than the span. In addition, registration is a measure of short-term memory that is preserved even in moderate dementia [25]. Recall is underscored in many of these tests. In the MMSE, it represents only 3 points ( $10 \%$ of the total score), this is quantitatively a low weighting for such an important consideration compared to the presumed importance of memory impairment in the early stages of dementia.

- A good number of existing dementia screening instruments use reading (MMSE, Mattis, Addenbrooke's Cognitive Examination Revised), writing (MMSE, 3MS, and Mattis), and/ or drawing tasks (MMSE, 3MS, Mini-Cog, MoCA, Mattis, RUDAS, NCSE, and CERAD Neuropsychological Instrument) that are irrelevant to illiterate subjects who represent the majority of the elderly population especially in Africa, Asia, and Latin America [see UNESCO Institute for Statistics, 26]. For instance, how do you interpret the poor graphic reproduction of a person who has never use a pen in their life? To include reading, writing, and drawing task scores in the assessment of dementia may produce false positives by inappropriately inflating the total scores.

- Strangely enough, dementia screening instruments neglect the assessment of executive functions (MMSE, Mini-Cog, RUDAS, Short Portable Mental Status Questionnaire, and the NCSE) that are essential for the diagnosis of frontotemporal dementia and subcortical dementia among others. These tests lag behind the current developments in our knowledge base of dementia.

The aforementioned limitations of the existing dementia screening tests argue for the development of a new screening tool to address these issues. We consider the instrument we are proposing, the Dementia Screening Battery-100 (DSB-100), as more effective in the detection of dementia in Tunisian elderly since it addresses and corrects all of the abovementioned issues, and its items and administration format are derived from the local cultural background. Indeed, cultural appropriateness provides more reliability to the inferences drawn from the test scores and the interpretations of differences in the patterns of the scores.

In this study, we present the DSB-100 and the rationale behind its development. This instrument is an analytic battery, composed of 10 subtests, developed and derived from established neuropsychological procedures, targeting memory, executive functions, praxia, language, and attentional and visuospatial deficits. The developed instrument attempts to screen dementia and type cognitive dysfunctions among the elderly Tunisian population who is mostly illiterate. Additionally, other goals of the current study were also to verify the impact of sociodemographic factors (sex, age, and education) on the DSB-100 scores and to examine its construct validity and its reliability. 
Bellaj et al.: Dementia Screening Battery-100

\section{Method}

The DSB-100

The DSB-100 is a test battery consisting of 10 subtests, which assess memory, executive functions, praxia, language, and attention and visuospatial functions. Its administration requires, on average, $15-20 \mathrm{~min}$.

Of note, the DSB-100 uses tasks that are classically known to be closely associated with attentional processes namely forward and backward span, even though the latter also engages working memory processes [27]. For these tasks, we opted to use names for forward and backward span. I.e., we present series of names, saying that we are reading male or female names as if they represent brothers or sisters from the same family. The task is to repeat the names immediately from the oldest to the youngest (Forward Names Span) - respecting the order of presentation - or from the youngest to the oldest (Backward Names Span). The use of names instead of digits removes the resistance found in illiterate individuals. Indeed, illiterates often complain that they cannot complete the forward or backward span task because they are not well-grounded in maths, even though the task does not call for it. The names span task appears more ecological, as it is similar to real world activities in which we say or repeat siblings' names in ascending or descending order.

We assessed language using 2 subtests: the comprehension of simple to complex orders and the naming of different parts of the body. These activities are important to assess receptive and expressive language processing. For the comprehension task, we selected, from the Token Test [28], 10 items of progressive difficulty. The most simple orders start with a verb, an adjective, and a noun. On the next higher level, we add "size" to the instructions. Then, the subjects must take into account the form and the color of 2 items. Next, we ask for the size, the color, and the form of 2 items. For the most complex level of the task, instructions include various suffixes, conjunctions, and adverbs. For the DSB-100 naming subtest, we know that all cultures and languages have specific names for parts of the human body [29]. In the naming task, we ask the subjects to name 10 parts of the body pointed at or touched by the tester (ears, teeth, nails, neck, shoulder, forehead, eyebrow, chin, elbow, and the index finger). Naming these body parts provides subjects with a range of difficulties - going from the eyes, nose, etc. to the eyebrows, nail, etc. [30]. On top, this task does not require any additional material (e.g., images and objects).

We assessed the subjects' visuospatial capacities through a modified version of the LineObject Orientation Judgment Test [31]. The test consists of 10 items with different levels of difficulty. It is a motor-free measure of the ability to estimate angular relationships between line segments by visually matching angled line pairs. The stimuli appear on the upper half of the page, and at the bottom of the page, there is an array of 11 lines, arranged in a semi-circle and pointing to a picture of an object. For each item, the participants are randomly provided with a set of two lines, and the task is to identify the object each line leads to.

We examined memory by presenting the participants an A4 page containing pictures of 10 objects, selected from the Snodgrass and Vanderwart database [11]. These pictures represent a mix of semantic living (animals, fruits, and vegetables) and non-living categories (furniture, clothing, school furniture, toys, tools, kitchen utensils, and transportation). The items are neither prototypic nor rare. They are ordered from 6 to 20 in each corresponding category frequency table. The items are also chosen considering naming agreement, visual complexity, and familiarity. After presenting the picture, we invite the individuals to name and memorize each picture. This procedure ensures a deeper semantic encoding strategy and activates the explicit memory system. After an interfering activity (digit span), the subjects are requested to recall the presented items. Then, a recognition task is proposed. For this task, a card with 40 pictures is presented, and the individuals have to decide which pictures have 
been shown in the learning phase. This card contains the 10 target items and 30 distractors (10 not related to the target items either semantically or formally; 10 semantically related, i.e., they are prototypes of the categories; and 10 formally related, i.e. they have almost the same form). Comparing performance in recall and in recognition is very useful for the understanding of memory-processing defects in dementia. The analysis of error types in recognition is also valuable.

We assessed executive functions through 2 tasks: the cognitive estimation task and the category verbal fluency task. The first is based on a well-known theoretically and experimentally validated test developed by Shallice and Evans [32]. The second is classically used in neuropsychological assessment as part of executive function evaluation [26]. For this purpose, we prefer a letter fluency task but used a semantic fluency test, since the latter is less sensitive to educational level and school experience [33].

We examined constructive apraxia, which is the type of apraxia most sensitive to brain lesions [34] using a short version of the "Stick construction task" developed by Butters and Barton [35], in which subjects are invited to put sticks together to reproduce 2-dimensional geometric constructions.

With its 10 subtests, the DSB-100 attempts to screen dementia and to type cognitive dysfunctions in individuals including those with little or no formal education. The goal of the current study was to present the DSB-100, the rationale behind its construction, and to examine its construct validity and reliability after verifying and correcting for the impact of sociodemographic factors namely sex, age, and education on its scores.

\section{Participants}

The study was conducted in Tunisia, North Africa. Two groups of participants took part in this study: a group diagnosed as demented and a group of healthy controls. The first group consisted of 42 demented patients; 27 female and 15 male. The mean age of this group was 69.38 years (SD $=7.75)$, and the mean number of years of education was $5.93(\mathrm{SD}=6.84)$. All met the DSM-IV criteria for dementia [22]. The diagnosis of dementia was taken by a panel discussion including neurologists and neuropsychologists according to gold standards based on a complete medical and neurological exam, a neuropsychological testing of major cognitive and conative functions, an objective assessment of daily life activities using an adapted version of the Instrumental Activities of Daily Living [36], biological exams (ApoE, hemocysteine, vitamin $\mathrm{B}_{12}$, folate, etc.) and a CT scan, MRI, or SPECT imaging. The demented participants were outpatients recruited from the Memory Center of the Department of Neurology at Razi Hospital in Tunis, Tunisia. Among the patients, 26 were diagnosed as having mild dementia ( $C D R=1)$ and 16 as moderate $(C D R=2)$. Patients having very mild/uncertain dementia $(\mathrm{CDR}=0.5$ ) or very severe dementia ( $C D R>3$ ) were excluded from the analysis of the current study, in addition to all patients having other chronic neurological conditions such as epilepsy, multiple sclerosis, patients living in nursing homes, or patients having significant hearing, sight, or motor deficits, and those with any combination of the above that may interfere with neuropsychological testing.

Healthy matched controls were 159 subjects; 81 female and 78 male. The mean age was 67.80 years (SD = 7.25), and the mean number of years of education was $5.47(\mathrm{SD}=6.64)$. All subjects were examined by a physician for general health. All showed no cognitive impairment $(\mathrm{CDR}=0)$ and were independent in activities of daily living. Individuals having sensory, motor, or intellectual deficiencies or a personal history of a psychiatric disorder, addiction, or a central nervous system disorder were excluded from the study. The controls were relatives of the patients and normal healthy individuals living in normal non-institutional residence. Individuals who did not live anymore in their family setting or who lived permanently in elderly centers were also excluded. 
Bellaj et al.: Dementia Screening Battery-100

Table 1. Demographic and general neuropsychological data of the dementia patients and healthy controls

\begin{tabular}{|c|c|c|c|}
\hline & $\begin{array}{l}\text { Dementia patients } \\
(n=42)\end{array}$ & $\begin{array}{l}\text { Healthy controls } \\
(n=159)\end{array}$ & $p$ value \\
\hline \multicolumn{4}{|l|}{ Sex } \\
\hline Female, $n(\%)$ & 27 (64.29\%) & $81(50.94 \%)$ & \multirow[t]{2}{*}{0.123} \\
\hline Male, $n(\%)$ & $15(35.71 \%)$ & $78(49.06 \%)$ & \\
\hline \multicolumn{4}{|l|}{ Age, years } \\
\hline Mean \pm SD & $69.38 \pm 7.75$ & $67.80 \pm 7.25$ & \multirow[t]{2}{*}{0.218} \\
\hline Min-max & $55-89$ & $55-90$ & \\
\hline \multicolumn{4}{|l|}{ Education, years } \\
\hline Mean \pm SD & $5.93 \pm 6.84$ & $5.47 \pm 6.64$ & \multirow[t]{2}{*}{0.645} \\
\hline Min-max & $0-21$ & $0-21$ & \\
\hline A-MMSE & $23.65 \pm 3.57$ & $28.14 \pm 1.53$ & 0.001 \\
\hline A-FAB & $10.59 \pm 2.55$ & $15.86 \pm 1.48$ & 0.001 \\
\hline A-GDS & $12.65 \pm 6.98$ & $6.91 \pm 3.92$ & 0.001 \\
\hline
\end{tabular}

As illustrated in Table 1, no significant differences were observed between the groups in terms of $\operatorname{sex}\left(\chi^{2}(1)=2.37, p=0.123\right)$, age $(t(199)=1.23, p=0.218)$, and education $(t(199)=$ $0.46, p=0.645)$.

\section{Procedure}

All participants underwent a structured interview to obtain demographic data, a detailed personal and family medical history, and information on alcohol or drug consumption. For patients with dementia, all required information was collected from relatives if needed. Cognitive tests were administered individually by clinical neuropsychologists after obtaining informed consent. The DSB-100 was administered as part of a larger neuropsychological assessment including the A-MMSE [37], the A-GDS [38], and the A-FAB [39] to have additional indices on the severity of patients' dementia, depression, and potential frontal dysfunctions. The same information was also used for comparisons with DSB-100 scores.

\section{Statistical Analysis}

To examine the effects of demographic variables on performance, we used a multiple linear regression analysis where the DSB-100 total score was the dependent variable and sex, age, and education were considered as predictors (independent variables). The Student $t$ test was used to compare the groups on demographic variables or overall difference in their DSB-100 scores. A MANOVA was also conducted to compare the group differences in the DSB-100 subtests followed by omnibus univariate tests. $\eta^{2}$ was used for measuring effect size, and the significance level was set at $p<0.05$.

To validate the DSB-100, we opted for the construct validation rather than other forms in the absence of any other available concurrent measure. Construct validity was done using principal component factor analysis (PCFA) with varimax rotation. Factor extraction was determined using a minimum eigenvalue of 1 .

To determine reliability, 2 types of coefficients were used: internal consistency using Cronbach's alpha coefficient and test-retest reliability using the Pearson Product Moment 
Table 2. Univariate analysis of the DSB-100 subtest performance in the dementia patients and healthy controls

\begin{tabular}{lcccc}
\hline Subtest & $\begin{array}{l}\text { Dementia patients } \\
(n=42)\end{array}$ & $\begin{array}{l}\text { Healthy controls } \\
(n=159)\end{array}$ & $p$ value & $\eta^{2}$ \\
\hline Forward Names Span & $3.95(1.27)$ & $5.47(1.12)$ & 0.001 & 0.19 \\
Backward Names Span & $2.59(0.99)$ & $4.18(1.24)$ & 0.001 & 0.18 \\
Recall & $4.76(0.87)$ & $7.08(1.69)$ & 0.001 & 0.28 \\
Recognition & $6.24(1.16)$ & $8.92(1.25)$ & 0.001 & 0.42 \\
Verbal Fluency & $4.90(1.61)$ & $8.72(1.39)$ & 0.001 & 0.57 \\
Comprehension & $7.57(1.71)$ & $9.63(0.79)$ & 0.001 & 0.34 \\
Naming & $7.24(1.28)$ & $9.56(0.80)$ & 0.001 & 0.39 \\
Cognitive Estimation & $5.29(1.67)$ & $8.39(1.53)$ & 0.001 & 0.38 \\
Line-Object Orientation Judgment & $4.26(1.38)$ & $5.93(1.75)$ & 0.001 & 0.11 \\
Constructive Praxia & $5.64(1.41)$ & $8.84(1.50)$ & 0.001 & 0.39 \\
\hline Total DSB-100 & $52.45(5.79)$ & $76.74(7.54)$ & 0.001 & 0.74 \\
\hline
\end{tabular}

All values are represented as means (SD).

Correlation Coefficient, where 27 patients and 34 healthy controls were retested by the same clinician 4-6 weeks after the initial assessment.

\section{Results}

The Kolmogorov-Smirnov test showed normal DSB-100 distribution for the healthy controls $\left(D_{\max }=0.075, p>0.20\right)$ and for the demented patients $\left(D_{\max }=0.105, p>0.20\right)$. Table 2 presents means and standard deviations for each group and each subtest. For both groups, the highest scores were seen in the Comprehension, Naming, and Recognition subtests. The poorest scores were seen in the Backward Names Span followed by the Forward Names Span, and Line-Object Orientation Judgment subtests.

For the total DSB-100 data, the overall multivariate test (Wilk's $\lambda)$ was significant $(F(10$, $190)=62.49, p<0.001, \eta^{2}=0.76$ ). Using the adjusted A-GDS score as a covariate in the multivariate analysis slightly attenuated the effect size, but it did not change the pattern of the results $\left(F(10,190)=44.48, p<0.001, \eta^{2}=0.74\right)$. For the MANOVA, omnibus univariate tests were significant $(p<0.001)$ for all subtests. Effect sizes ranged from 0.11 for the Line-Object Orientation Judgment subtest to 0.57 for the Verbal Fluency subtest. Table 2 presents the means and standard deviations by group along with significance tests and effect sizes for each subtest.

\section{Effects of Demographic Variables on the DSB-100}

The effect of demographic variables (sex, age, and education) on the DSB-100 scores were analyzed by step-wise multiple linear regression analysis. The results indicated that, while the DSB-100 score was significantly sensitive to age and education $(p<0.001)$, it was not affected by sex $(p=0.262)$. The original scores of the DSB-100 should be adjusted for age and education: final score $=82.05+[($ number of years of education $\times 0.778)+($ age $\times-0.160)]$.

Regression analysis retains "age" and "education" as variables with significant importance, while "sex" was excluded as insignificant. The results in Table 3 show the value of $R^{2}$ and suggest that the percentage of variance explained by the 2 sociodemographic variables 
Table 3. Relationship between the total score of the DSB-100 and the sociodemographic variables: value of standardized $\beta$ coefficients and coefficients of determination $R^{2}$

\begin{tabular}{|c|c|c|c|c|c|c|c|}
\hline \multirow[t]{2}{*}{ Predictors } & \multicolumn{4}{|l|}{ Coefficients } & \multicolumn{3}{|l|}{ Model } \\
\hline & $\begin{array}{l}\text { non-standardized } \\
\beta\end{array}$ & $\begin{array}{l}\text { standardized } \\
\beta\end{array}$ & $\begin{array}{l}\text { Student } \\
t \text { test }\end{array}$ & $p$ value & $\begin{array}{l}F \\
\text { (ANOVA) }\end{array}$ & $p$ value & $R^{2}$ \\
\hline Constant & 82.05 & - & & & 145.59 & 0.0001 & 0.64 \\
\hline Sex & & 0.005 & 0.96 & 0.924 & & & \\
\hline Education & 1.09 & 0.778 & 16.41 & 0.0001 & & & \\
\hline Age & -0.166 & -0.160 & -3.37 & 0.001 & & & \\
\hline
\end{tabular}

Table 4. Values of corrections to the initial scores to obtain adjusted total scores

\begin{tabular}{|c|c|c|c|c|c|c|c|}
\hline & \multicolumn{7}{|c|}{ Age, years } \\
\hline & $55-57$ & $58-63$ & $64-69$ & $70-74$ & $75-80$ & $81-86$ & $\geq 87$ \\
\hline \multicolumn{8}{|c|}{ Education, years } \\
\hline $0-1$ & 1 & 2 & 3 & 4 & 5 & 6 & 7 \\
\hline $2-3$ & 0 & 1 & 2 & 3 & 4 & 5 & 6 \\
\hline $4-5$ & -1 & 0 & 1 & 2 & 3 & 4 & 5 \\
\hline $6-7$ & -2 & -1 & 0 & 1 & 2 & 3 & 4 \\
\hline $8-9$ & -3 & -2 & -1 & 0 & 1 & 2 & 3 \\
\hline $10-11$ & -4 & -3 & -2 & -1 & 0 & 1 & 2 \\
\hline $12-13$ & -5 & -4 & -3 & -2 & -1 & 0 & 1 \\
\hline $14-15$ & -6 & -5 & -4 & -3 & -2 & -1 & 0 \\
\hline $16-17$ & -7 & -6 & -5 & -4 & -3 & -2 & -1 \\
\hline $18-19$ & -8 & -7 & -6 & -5 & -4 & -3 & -2 \\
\hline $20-21$ & -9 & -8 & -7 & -6 & -5 & -4 & -3 \\
\hline
\end{tabular}

age and education was $64 \%$. The DSB-100 performance depends heavily on age and education. Thus, the scores obtained by the DSB-100 must be adjusted according to the relative weight of the significant demographic variables, namely age and education. For this purpose, we ran a calculation using the multiple linear regression of the quantitative impact of the variables age and education on the total DSB-100 score. Table 4 provides the suggested appropriate corrections to adjust the observed total scores by adding the necessary correction (positive or negative) based on age and education level of the participants.

Hereafter, we provide 3 examples for the use of the correction table (Table 4). For instance, for an illiterate 67-year-old man with a total observed score of 60 on the DSB-100, 3 points must be added to his initial score to arrive at the adjusted score where the effects of age and education are taken into account. His adjusted score is 63 . Another example, for a 55 -year-old woman who had had only 2 years of education and who obtained 87 points on the DSB-100, we keep the score as it is because the correction value from Table 4 is 0 . A last example, for a woman aged 60, with a Master of Science (equivalent to 17 years of education) and a score of 75 on the DSB-100, we need to deduct 6 points from the total score, so her adjusted score is 69 .

When we applied a multiple regression analysis to each DSB-100 subtest, the results revealed that the effect of education was highly significant for all subtests, while the effect of age was merely significant for the Constructive Praxia $(p<0.001)$ and Recognition $(p<0.05)$ 
Table 5. Standardized scores for the effects of sex, age, and education on the DSB-100 subtest scores

Bellaj et al.: Dementia Screening Battery-100

\begin{tabular}{lrll}
\hline Subtests and total DSB-100 & Sex & Age & Education \\
\hline Forward Digit Span & $0.151^{*}$ & -0.115 & $0.588^{* * *}$ \\
Backward Digit Span & 0.118 & -0.066 & $0.574^{* * *}$ \\
Recall & -0.043 & -0.024 & $0.582^{* * *}$ \\
Recognition & -0.034 & $-0.186^{*}$ & $0.357^{* * *}$ \\
Verbal Fluency & -0.140 & -0.032 & $0.244^{* *}$ \\
Comprehension & -0.110 & -0.120 & $0.215^{* *}$ \\
Naming & -0.080 & -0.138 & $0.254^{* * *}$ \\
Cognitive Estimation & 0.042 & -0.036 & $0.185^{*}$ \\
Line-Object Orientation Judgment & 0.042 & -0.059 & $0.870^{* * *}$ \\
Constructive Praxia & -0.026 & $-0.263^{* * *}$ & $0.382^{* * *}$ \\
\hline \multirow{2}{*}{$* p<0.05, * * p<0.01,{ }^{* * *} p<0.001}$. & & \\
\hline
\end{tabular}

Table 6. Varimax with a Kaiser normalization-rotated component matrix for the DSB-100 items in the healthy control group

\begin{tabular}{|c|c|c|c|c|}
\hline \multirow[t]{2}{*}{ Subtests } & \multicolumn{4}{|l|}{ Factors } \\
\hline & $\begin{array}{l}\text { Attentional } \\
\text { and Visuospatial }\end{array}$ & Memory & Language & $\begin{array}{l}\text { Executive } \\
\text { Functions }\end{array}$ \\
\hline Line-Object Orientation Judgment & 0.84 & 0.08 & -0.06 & 0.05 \\
\hline Forward Names Span & 0.83 & 0.10 & -0.01 & 0.02 \\
\hline Backward Names Span & 0.82 & 0.21 & 0.18 & 0.13 \\
\hline Constructive Praxia & 0.47 & 0.36 & 0.06 & 0.24 \\
\hline Recall & 0.11 & 0.82 & 0.03 & -0.01 \\
\hline Recognition & 0.20 & 0.76 & -0.02 & 0.02 \\
\hline Comprehension & 0.05 & 0.10 & 0.84 & 0.09 \\
\hline Naming & 0.01 & -0.06 & 0.82 & 0.06 \\
\hline Cognitive Estimation & 0.02 & 0.15 & -0.04 & 0.84 \\
\hline Verbal Fluency & 0.21 & -0.14 & 0.28 & 0.72 \\
\hline Explained variance, \% & 29.96 & 15.92 & 11.09 & 10.08 \\
\hline
\end{tabular}

subtests (Table 5). An effect of sex was observed only for the Forward Digit Span subtest scores $(p<0.05)$.

\section{Validity of the DSB-100}

To validate the DSB-100, we opted for the construct validation, which is considered the most appropriate for adapted or newly developed instruments. Construct validity was done using PCFA on the healthy control group's adjusted scores.

The performed exploratory PCFA using eigenvalues $>1$ yielded a 4 -factor solution. Subjected to an orthogonal rotation (varimax), the 4 extracted factors accounted for $67.05 \%$ of the total variance with $29.96,15.92,11.09$, and $10.08 \%$, respectively. The factors can be labeled as: (a) "Attentional and Visuospatial", composed of efficiency measures on the LineObject Orientation Judgment, Forward Names Span, Backward Names Span, and Constructive Praxia subtests, (b) "Memory", defined by high loadings for the Recall and Recognition subtests, (c) "Language", based on the scores of the Comprehension and Naming subtests, and (d) "Executive Functions", consisting of high loadings on the Cognitive Estimation and Verbal Fluency subtests (Table 6). 
Table 7. Item characteristics of the DSB-100

\begin{tabular}{ll}
\hline Items & $\begin{array}{l}\text { Cronbach's alpha } \\
\text { if item is deleted }\end{array}$ \\
\hline Forward Names Span & 0.72 \\
Backward Names Span & 0.72 \\
Recall & 0.71 \\
Recognition & 0.73 \\
Verbal Fluency & 0.76 \\
Comprehension & 0.75 \\
Naming & 0.75 \\
Cognitive Estimation & 0.76 \\
Line-Object Orientation Judgment & 0.69 \\
Constructive Praxia & 0.73 \\
\hline Total DSB-100 score & 0.76
\end{tabular}

Reliability of the DSB-100

As illustrated in Table 7, the internal consistency of the DSB-100, obtained by Cronbach's alpha for the healthy controls, was acceptable $(\alpha=0.76)$. This indicates that all items measure the same construct without redundancy.

We calculated the test-retest reliability by re-testing 27 patients and 34 controls, by the same clinician, 4-6 weeks after the initial assessment. The test-retest correlation coefficients for the total DSB-100 score calculated for each sample were high for patients $(r=0.81, p<$ $0.001)$ and for healthy matched controls $(r=0.87, p<0.001)$. This indicates that performance on the DSB-100 is stable over time.

\section{Discussion}

The first objective of this study was to introduce the DSB-100 and to present the rationale behind its construction. This new dementia screening battery was designed to address shortcomings found in existing dementia screening instruments (such as the MMSE, Mattis, MoCA, or 3MS) by including more accurate assessments of long-term memory, an ecological assessment of short-term and working memory, a contextualized assessment of visuospatial functions, an assessment of executive functions, and the inclusion of less culturally biased procedures and instrument contents. The second objective was to assess the effect of sociodemographic variables, namely sex, age, and education, on the battery scores and to examine the instrument's psychometric properties.

Concerning the rationale behind the construction of the DSB-100, 2 main reasons motivated its conception. The first sought to address fundamental issues when using existing dementia screening tools in non-Western cultures. The second sought to bridge the gap between the rapidly changing knowledge in the field of dementia and the validity of dementia screening tools. To this effect, the proposed instrument sought to correct biases of cultural nature in available dementia screening instruments and to address methodological issues arising from the interplay of both the fundamental and cultural factors combined. These problems affect the assessment of dementia and called for the construction of a new screening instrument to overcome the many limitations of widely used dementia screening tools.

There are 2 main shortcomings of fundamental nature with the available dementia screening tools: they miss some major functions, and they do not detect early stages of 
dementia. Currently, there is a clear incongruity between the psychological functions tapped in existing screening tools and the evolving diagnostic criteria of dementia. For instance, executive functions are a major criterion for dementia, ever since the publication of the DSM IV [22], and are currently critically important diagnostic criteria of dementia. However, they are not measured in widely used dementia screening instruments such as the MMSE and 3MS. False negatives can easily occur for patients suffering from frontotemporal dementia, corticobasal dementia, vascular dementia, and subcortical dementia. In addition, existing screening tools do not dissociate between recall and recognition in long-term memory tasks, even though it is important to distinguish between cortical and subcortical dementias [40]. Available screening instruments offer just gross measures of visuoconstructive functions and do not dissociate between visuospatial and visuoconstructive deficits - that can be helpful in differentiating between Lewy Body dementia and Alzheimer disease [41]. Screening tools should be able to detect dementia as early as possible to enable early intervention, rehabilitation, and disease management. Nevertheless, most of the existing screening tools (e.g., the MMSE, MoCA, and Mattis) seem to serve rather as a severity measure than an early detection tool. For instance, the MMSE devotes one third of its total score to temporal and spatial orientation, alterations happen here at later stages of dementia, while it fails to detect executive and working memory dysfunctions, which occur in the early stages of many types of dementia [42-44].

Existing dementia screening instruments are notorious for overlooking important cultural dimensions and for including biases that affect the proper assessment of dementia and, thus, its detection [45]. The development of a culturally sensitive and appropriate instrument has the potential to reduce assessment biases and interpretation errors. Studies in cultural neuroscience offer proof of cultural differences in brain activation patterns. Such differences were observed in both lower-level processes [46-50] as well as higher-order ones [51-54]. Findings argue for a brain architecture that is not universal (for a review, see Caramazza [55]) despite the fact that the brain works according to identical laws [56]. The aforementioned arguments represent findings from studies on brain-injured illiterate patients [30,57], brain-injured bilinguals [57], and evidence from experimental neuroscience with normal monolinguals and bilinguals [58]. All testify that differences observed in performance in cognitive style do not vary only according to biological factors, the nature and extent of brain lesion, laterality, sex, and age. These differences also depend on cultural factors that arrange cerebral organization itself. Among these factors, we noted the effect of the level of education, bilingualism, and the importance of the type of the script used to write on brain organization.

In addition to the importance of the impact of cultural factors on cognitive functioning, demographic specificities are well-established determinants of cognitive performance. Knowing specific demographic facts is essential to conceptualize and to build any health program for dementia. African countries, for example, are characterized by a high level of fertility, a high level of multiculturalism, and a high level of illiteracy [59]. By 2050, the worldwide population over 60 years will reach two billion people, three quarters of which will be from African countries. These countries are the hallmark of multicultural, multilingual, and multiethnic societies and have the highest rates of illiteracy in the world [60]. For instance, in Tunisia, more than $85 \%$ of all individuals older than 60 years are illiterate [61], and the country is predominantly multicultural. Throughout history, the indigenous Berber culture of Tunisia has been influenced by multiple invading civilizations including the Carthaginians, Romans, Vandals, Bezants, Africans, Arabs, Turkish, Spanish, and French, but the current main cultural influences remain Arabic and Islamic [62]. Besides, the sociolinguistic context is quite complex, since Phoenician, Latin, Arabic, Hebrew, Italian, Spanish, Turkish, and French have been added to the Berber basis [62]. 
In the Western world, illiteracy is not generally a problem among the elderly [63]. However, in non-Western and low-to-middle-income countries such as Tunisia, illiteracy continues to be characteristic of elderly people especially women [26]. Existing dementia screening tools assess writing, reading, calculating, and drawing. When the assessed elderly is "culturally different" and illiterate like it is the case for many people in African, Asian and South-American countries, existing dementia screening tools do not only contain cultural biases but are inherently inappropriate as screening tools. To continuously use them would systematically introduce methodological issues and fundamental diagnostic errors resulting in high rates of false positives [64]. Adapting the DSB-100 to the Tunisian context produced a culturally appropriate measure that has a great potential for application in similar contexts (such as elderly from developing countries of non-Western cultures and from countries with low literacy levels; the DSB-100 may also be the instrument of choice with immigrants from developing countries of non-Western cultures residing in Western countries). The DSB-100 has also the potential to be easily adapted to many other cultures (Western and non-Western) with straightforward modifications to some of its subscales. It simply requires the inclusion of verbal and pictorial stimuli that are equivalent to the DSB-100 items, based on local standardized databases similar to Battig and Montague's verbal categorical database [10] and Snodgrass and Vanderwart's pictorial database [11].

In addition to the importance of the impact of cultural factors no cognitive functioning, demographic specificities are well-established determinants of cognitive performance [64]. The study findings outlined the sensitivity of the DSB-100 to some sociodemographic variables. It revealed that besides all the efforts to select and develop tasks that are applicable to illiterate and low-educated individuals, the influence of education remains significant. Tasktaking behavior may inherently contribute to lowering test performance in illiterate individuals, as they tend to perform poorly on tests due to a discernible lack of training and clear deficiencies in test taking [65]. Illiteracy in itself affects cognitive capacities (for a review, see Julayanont and Ruthirago [66]), and this may explain why the educational effect was significant in all tasks, even if the task acceptability and instruction comprehension were fine. The adjustment of obtained data may, in part, solve the problem. Barona et al.'s adjustment technique [67] was useful to attenuate general education and age effects, even though it may be criticized, since it presumes a linearity of the effect of education and age on cognitive performance [68].

The study was conducted in Tunisia, where Tunisian, an Arabic dialect, is spoken. Since Tunisia is a developing country, we selected 55 years of age as our lower bound - as was the case for Chinese [69] or Brazilian populations [70]. The DSB-100 displayed good psychometric properties. The total score's reliability concerning its stability was high in the testretest method and quite satisfactory regarding its internal homogeneity. For the internal consistency of the DSB-100 subtests, Cronbach's alpha was acceptable in the healthy matched controls for all of the subtests

Factor analysis was a useful quantitative method to examine the dimensionality of the DSB-100. In fact, data reduction yielded 4 factors, namely "Attentional and Visuospatial", "Memory", "Language", and "Executive Functions", providing a starting point for clinicians in the diagnosis of dementia types. Evidence from the construct validity proved that the clinical utility of the DSB-100 was comparable to most widely used tests like the Mattis [16].

Thus, the DSB-100 is an instrument for the detection and the assessment of cognitive changes associated with dementia. It was constructed for a non-Western elderly population with low literacy/illiterate individuals living in a developing country. However, as noted previously, the instrument is easily adaptable to many other cultures (Western and nonWestern) with straightforward modifications to some of its subscales. This is especially the case as tasks composing this instrument were selected from various well-known neuropsy- 
chological tests. This study showed that the DSB-100 is a suitable, reliable, and valid tool for the screening of dementia. It yields information about 4 cognitive domains: attention and visuospatial functions, memory, language, and executive functions. It can also be useful to orient clinicians toward the type of dementia condition via the analysis of the cognitive deficit profile and to plan intervention treatment strategies. Possessing normative data and deciding about cutoff scores are essential to take diagnostic decisions and to apply the instrument to follow-up patients' change under cognitive rehabilitation and/or appropriate medication. Other aspects of the psychometric properties of the DSB-100 need to be examined through further studies, especially concurrent validity and clinical validity respecting different stages of severity of cognitive decline in different types of dementia.

\section{Acknowledgement}

We thank all patients who contributed to this research and their caregivers.

\section{Disclosure Statement}

The authors declare no conflict of interest.

\section{References}

1 Vos SJ, van Rossum, Verhey F, Knol DL, Soininen H, Wahlund LO, Hampel H, Tsolaki M, Minthon L, Frisoni GB, Froelich L, Nobili F, van der Flier W, Blennow K, Wolz R, Scheltens P, Visser PJ: Prediction of Alzheimer disease in subjects with amnestic and non-amnestic MCI. Neurology 2013;80:1124-1132.

2 Brown DE: Human universals, human nature, human culture. Daedalus 2004;133:47-54.

3 Nisbett RE, Norenzayan A: Culture and cognition; in Pashler H, Medin D (eds): Stevens' Handbook of Experimental Psychology: Memory and Cognitive Processes, ed 3. New York, John Wiley \& Sons, 2002, vol 2, pp 561-597.

4 Heaton RK, Ryan L, Grant I, Matthews CG: Demographic influences on neuropsychological test performance; in Grant I, Adams KM (eds): Neuropsychological Assessment of Neuropsychiatric Disorders, ed e. New York, Oxford University Press, 1996, pp 141-163.

5 Malgady RG: The question of cultural bias in assessment and diagnosis of ethnic minority clients: let's reject the Null Hypothesis. Prof Psychol Research Pract 1996;27:73-77.

6 Bellaj T, Le Gall D: L'évaluation neuropsychologique du Maghrébin; in Amieva H, Belin C, Maillet D (eds): L'évaluation neuropsychologique : de la norme à l'exception. Marseille, De Boeck-Solal, 2016.

7 Hambleton RK, Merenda PF, Spielberger CD: Adapting Educational and Psychological Tests for Cross-Cultural Assessment. London, Lawrence Erlbaum Associates, 2005.

-8 Van de Vijver F, Tanzer NK: Bias and equivalence in cross-cultural assessment: an overview. Eur Rev Appl Psychol 2004;54:119-135.

9 De Mattos Pimenta Parente MA, Lecours AR: L'influence des facteurs culturels en neuropsychologie et neurolinguistique. Rev Int Sci Soc 1988;115:109-122.

10 Battig WF, Montague E: Category norms for verbal items in 56 categories: a replication and extension of the Connecticut category norms. J Exp Psychol Monogr 1969;80:1-45.

$\checkmark 11$ Snodgrass JG, Vanderwart M: A standardized set of 260 pictures: norms for name agreement, image agreement, familiarity, and visual complexity. J Exp Psychol: Hum Learn Mem 1980;6:174-215.

12 Van de Vijver F, Hambleton RK: Translating tests: some practical guidelines. Eur Psychol 1996;1:89-99.

-13 Tanzer NK, Sim CQE: Adapting instruments for use in multiple languages and cultures: a review of the ITC Guidelines for Test Adaptations. Eur J Psychol Assess 1999;15:258-269.

14 Folstein MF, Folstein SE, McHugh PR: "Mini-mental state": a practical method for grading the cognitive status of patients for the clinician. J Psychiatr Res 1975;12:189-198.

15 Nasreddine ZS, Phillips NA, Bedirian V, Charbonneau S, Whitehead V, Collin I, Cummings JL, Chertkow H: The Montreal Cognitive Assessment, MoCA: a brief screening tool for mild cognitive impairment. J Am Geriatr Soc 2005;53:695-699.

16 Mattis S: Dementia Rating Scale: Professional Manual. Odessa, Psychological Assessment Resources, 1973. 
Pfeiffer E: A Short Portable Mental Status Questionnaire for the assessment of organic brain deficit in elderly patients. J Am Geriatr Soc 1975;23:433-441.

18 Teng EL, Chui HC: The modified Mini-Mental State (3MS) examination. J Clin Psychiatry 1987;48:314-318.

19 Mioshi E, Dawson K, Mitchell J, Arnold R, Hodges JR: The Addenbrooke's Cognitive Examination Revised (ACE-R): a brief cognitive test battery for dementia screening. Int J Geriatr Psychiatry 2006;21:1078-1085.

$>20$ Kiernan RJ, Mueller J, Langston JW, Van Dyke C: The Neurobehavioral Cognitive Status Examination, a brief but differentiated approach to cognitive assessment. Ann Intern Med 1987;107:481-485.

21 Morris JC, Mohs RC, Rogers H, Fillenbaum G, Heyman A: Consortium to establish a registry for Alzheimer's disease (CERAD) clinical and neuropsychological assessment of Alzheimer's disease. Psychopharmacol Bull 1988;24:641-652.

22 American Psychiatric Association: Diagnostic and Statistical Manual of Mental Disorders, ed 4. Washington, American Psychiatric Association, 1994.

23 Borson S, Scanlan JM, Chen PJ, et al: The Mini-Cog as a screen for dementia: validation in a population-based sample. J Am Geriatr Soc 2003;51:1451-1454.

24 Storey JE, Rowland JTJ, Conforti DA, Dickson HG: The Rowland Universal Dementia Assessment Scale (RUDAS): a multicultural cognitive assessment scale. Int Psychogeriatr 2005;16:13-31.

25 Baddeley A, Bressi S, Della Sala S, Logie R, Spinnler H: The decline of working memory in Alzheimer's disease. Brain 1991;114:2521-2542.

26 UNESCO Institute for Statistics: Adult and Youth Literacy: National, Regional and Global Trends, 1985-2015. Montreal, UNESCO Institute for Statistics, 2013. Available at: http://www.uis.unesco.org/Education/Documents/fs26-2013-literacy-en.pdf (accessed December 24, 2016).

27 Lezak M, Howieson D, Bigler E, Tranel D: Neuropsychological Assessment. New York, Oxford University Press, 2012.

28 De Renzi E, Vignola LA: The token test: a sensitive test to detect receptive disturbances in aphasics. Brain 1962;85:665-678.

29 Andersen ES: Lexical universals of body-part terminology; in Greenberg JH (ed): Universals of Human Language. Stanford, Stanford University Press, 1978, pp 335-368.

-30 Rosselli M, Ardila A, Rosas P: Neuropsychological assessment in illiterates. II. Language and praxic abilities. Brain Cogn 1990;12:281-296. Benton AL, Varney N, Hamsher K: Visuospatial judgment: a clinical test. Arch Neurol 1978;35:364-367.

32 Shallice T, Evans ME: The involvement of the frontal lobes in cognitive estimation. Cortex 1978;14:294-303.

-33 Mathuranath PS, George A, Cherian JP, Alexander A, Sarma SG, Sarma PS: Effects of age, education and gender on verbal fluency. J Clin Exp Neuropsychol 2003;25:1057-1064.

34 Le Gall D, Aubin G: L'apraxie. Marseille, Solal, 2003.

-35 Butters N, Barton M: Effect of parietal lobe damage on the performance of reversible operations in space. Neuropsychologia 1970;8:205-214.

36 Bellaj T, Ben Jemaa S, Attia-Romdhane N, Dhiffallah M, Ben Ali N, Bouaziz M, Mrabet A: Version arabe du Mini Mental State Examination (A-MMSE) : fidélité, validité et données normatives. Tunis Med 2008;86:768-776.

37 Bellaj T, Ben Jemaa S, Anane N, Attia-Romdhane N, Ben Youssef K, Kahouaji H, Bouaziz M, Mrabet A: Version arabe de la Geriatric Depression Scale (A-GDS): fidélité, validité et données normatives. Tunis Med 2008;86: 801-808.

38 Ben Jemaa S, Bellaj T, Attia-Romdhane N, Cherif A, Oudiaa Zakraoui N, Bouaziz M, Mrabet A: La Frontal Assessment Battery (FAB): fidélité, validité et étalonnage d'une forme Arabe. Tunis Med 2008;86:793-800

-39 Karantzoulis S, Galvin J-E: Distinguishing Alzheimer's disease from other major forms of dementia. Expert Rev Neurother 2011;11:1579-1591.

40 Li X, Rastogi P, Gibbons JA, Chaudhury S: Visuo-cognitive skill deficits in Alzheimer's disease and Lewy body disease: a comparative analysis. Ann Indian Acad Neurol 2014;17:12-18.

41 Baudic S, Barba GD, Thibaudet MC, Smagghe A, Remy P, Traykov L: Executive function deficits in early Alzheimer's disease and their relations with episodic memory. Arch Clin Neuropsychol 2010;21:15-21.

-42 Levy G, Jacobs DM, Tang M-X, Côté LJ, Louis ED, Alfaro B, Mejia H, Stern Y, Marder K: Memory and executive function impairment predict dementia in Parkinson's disease. Mov Disord 2002;17:1221-1226.

43 Torralva T, Roca M, Gleichgerrcht E, Bekinschtein T, Manes F: A neuropsychological battery to detect specific executive and social cognitive impairments in early frontotemporal dementia. Brain 2009;132:1299-1309.

44 Manly J, Espino D: Cultural influences on dementia recognition and management. Clin Geriatr Med 2004;20: 93-119.

45 Ansari D: Effects of development and enculturation on number representation in the brain. Nat Rev Neurosci 2008; 9:278-291.

-46 Gutchess AH, Welsh RC, Boduroglu A, Park DC: Cultural differences in neural function associated with object processing. Cogn Affect Behav Neurosci 2006;6:102-109.

-47 Ji LJ, Peng K, Nisbett RE: Culture, control, and perception of relationships in the environment. J Pers Soc Psychol 2000;78:943-955.

48 Kitayama S, Duffy S, Kawamura T, Larsen JT: Perceiving an object and its context in different cultures: a cultural look at new look. Psychol Sci 2003;14:201206.

49 Lin Z, Lin Y, Han S: Self-construal priming modulates visual activity underlying global/local perception. Biol Psychol 2008;77:93-97. 
-50 Chiao JY, Iidaka T, Gordon HL, Nogawa J, Bar M, Aminoff E, Sadato N, Ambady N: Cultural specificity in amygdala response to fear faces. J Cogn Neurosci 2008;20:2167-2174.

51 Kobayashi C, Glover GH, Temple E: Cultural and linguistic influence on neural bases of "Theory of Mind": an fMRI study with Japanese bilinguals. Brain Lang 2006;98:210-220.

$52 \mathrm{Ng} \mathrm{SH}$, Han S, Mao L, Lai JCL: Dynamic bicultural brains: fMRI study of their flexible neural representation of self and significant others in response to culture primes. Asian J Soc Psychol 2010;13:83-91.

53 Zhu Y, Zhang L, Fan J, Han S: Neural basis of cultural influence on self-representation. Neuroimage 2007;34: 1310-1316.

54 Han S, Ma Y: Cultural differences in human brain activity: a quantitative meta-analysis. Neuroimage 2014;99: 293-300.

-55 Caramazza A: On drawing inferences about the structure of normal cognitive systems from the analysis of patterns of impaired performance: the case for single-patient studies. Brain Cogn 1986;5:41-66.

56 Ardila A, Rosselli M, Rosas P: Neuropsychological assessment in illiterates: visuospatial and memory abilities. Brain Cogn 1989;11:147-166.

57 Galloway LM: Contributions of the Right Cerebral Hemisphere to Language and Communication Issues in Cerebral Dominance with Special Emphasis on Bilingualism, Second Language Acquisition, Sex Differences and Certain Ethnic Groups; doctoral thesis, University of California, Los Angeles, 1981.

58 Barton MI, Goodglass H, Shai A: Differential recognition of tachistoscopically presented English and Hebrew words in right and left visual fields. Percept Mot Skills 1965;21:431-437.

59 United Nations, Department of Economic and Social Affairs, Population Division: World Population Ageing 2013. Available at: http://www.un.org/en/development/desa/population/publications/pdf/ageing/WorldPopulationAgeing2013.pdf (accessed December 24, 2016).

60 Institut National de la Statistique : Données officielles relatives au recensement de 2004 effectué en Tunisie. Available at: http://www.ins.nat.tn/indexfr.php (accessed April 4, 2015).

61 Ben Rejeb R: Intelligence, test et culture. Paris, L'Harmattan, 2001.

62 UNESCO Institute for Statistics: 50th Anniversary of International Literacy Day: Literacy Rates Are on the Rise but Millions Remain Illiterate. UIS Fact Sheet, September 2016, No. 38, pp 1-10. Available at: http://www.uis. unesco.org/literacy/Documents/fs38-literacy-en.pdf (accessed December 19, 2016).

63 Daugherty JC, Puente AE, Fasfous AF, Ruzzante NH, Pérez-Garcia M: Diagnostic mistakes of culturally diverse individuals when using North American neuropsychological tests. Appl Neuropsychol Adult 2017;24:16-22.

-64 O’Bryant SE, O'Jile JR, McCaffrey RJ: Reporting of demographic variables in neuropsychological research: trends in the current literature. Clin Neuropsychol 2004;18:229-233.

65 Brucki SM, Nitrini R: Cancellation task in very low educated people. Arch Clin Neuropsychol 2008;23:139147.

-66 Julayanont P, Ruthirago D: The illiterate brain and the neuropsychological assessment: from the past knowledge to the future new instruments. Appl Neuropsychol Adult 2016 [Epub ahead of print].

67 Barona A, Reynolds CR, Chastain R: A demographically based index of premorbid intelligence for the WAIS-R. J Consult Clin Psychol 1984;52:885-887.

-68 Hawkins KA: Limitations to the validity of the Barona Regression Formula and similar demographically-based methods of estimating preinjury intellectual functioning. Behav Sci Law 1995;13:491-503.

69 Zhang MY, Katzman R, Salmon D, Jin H, Cai GJ, Wang ZY, Qu GY, Grant I, Yu E, Levy P, Klauber MR, Liu WT: The prevalence of dementia and Alzheimer's disease in Shanghai, China: impact of age, gender, and education. Ann Neurol 1990;27:428-437.

70 Porto CS, Fichman LC, Caramelli P, Bahia VS, Nitrini R: Brazilian version of the Mattis Dementia Rating Scale: diagnosis of mild dementia in Alzheimer's disease. Arq Neuropsiquiatr 2003;61:339-345. 